\title{
Balance training and ballistic strength training are associated with task-specific corticospinal adaptations
}

\author{
M. Schubert, ${ }^{1,2}$ S. Beck, ${ }^{1,4}$ W. Taube, ${ }^{3}$ F. Amtage, ${ }^{2}$ M. Faist ${ }^{2}$ and M. Gruber ${ }^{3}$ \\ ${ }^{1}$ Spinal Cord Injury Centre, University Hospital Balgrist, Zürich, Switzerland \\ ${ }^{2}$ Department of Clinical Neurology and Neurophysiology, University of Freiburg, Germany \\ ${ }^{3}$ Department of Sport Science, University of Freiburg, Germany \\ ${ }^{4}$ Human Motor Control Section, NINDS, National Institutes of Health, Bethesda, MD, USA
}

Keywords: human, plasticity, posture, TMS, training

\begin{abstract}
The aim of this study was to investigate the role of presumably direct corticospinal pathways in long-term training of the lower limb in humans. It was hypothesized that corticospinal projections are affected in a training-specific manner. To assess specificity, balance training was compared to training of explosive strength of the shank muscles and to a nontraining group. Both trainings comprised 16 1 - $\mathrm{h}$ sessions within 4 weeks. Before and after training, the maximum rate of force development was monitored to display changes in motor performance. Neurophysiological assessment was performed during rest and two active tasks, each of which was similar to one type of training. Hence, both training groups were tested in a trained and a nontrained task. H-reflexes in soleus (SOL) muscle were tested in order to detect changes at the spinal level. Corticospinal adaptations were assessed by colliding subthreshold transcranial magnetic stimulation to condition the SOL H-reflex. The short-latency facilitation of the conditioned $\mathrm{H}$-reflex was diminished in the trained task and enhanced in the nontrained task. This was observable in the active state only. On a functional level, training increased the rate of force development suggesting that corticospinal projections play a role in adaptation of leg motor control. In conclusion, long-term training of shank muscles affected fast corticospinal projections. The significant interaction of task and training indicates context specificity of training effects. The findings suggest reduced motor cortical influence during the trained task but involvement of direct corticospinal control for new leg motor tasks in humans.
\end{abstract}

\section{Introduction}

Strength and balance training play an important role in sports and neurological rehabilitation. Neural plasticity contributes to early adaptive effects seen within the first weeks of training. However, the sites of neural adaptation, their interactions and mechanisms are still largely unknown. In order to rehabilitate patients with central nervous lesions such as stroke or spinal cord injury, it is important to understand whether a particular training preferentially induces plastic changes in spinal or cortical sensory-motor circuits.

Brain imaging as well as electrophysiological studies have provided evidence for primary motor cortex plasticity during the early phase of motor skill acquisition (Pascual-Leone et al., 1994, 1995; Classen et al., 1998; Muellbacher et al., 2001, 2002; Lotze et al., 2003a; Puttemans et al.,2005). These studies demonstrated increased cortical excitability or enlargement of upper limb representational area. Increased motor excitability was also observed during lower limb skill acquisition, suggesting similar mechanisms in upper and lower extremities (Schubert et al., 1999; Perez et al., 2004, 2006; Taube et al., 2007). Besides cortical adaptations, spinal plasticity is also involved in optimizing motor behaviour (Wolpaw \& Lee, 1989; Remple et al., 2001; Meunier et al., 2007a,b). Moreover, motor training has been reported to induce an increase in electroencephalogram (EEG)-electromyogram (EMG) coherence, suggesting that the

Correspondence: Dr M. Schubert, 'Spinal Cord Injury Centre, as above.

E-mail: martin.schubert@balgrist.ch modulation of projections between motor cortex and spinal circuitry is relevant (Perez et al., 2006). However, little is known about how motor cortex interacts with the spinal cord during training and whether corticospinal drive is modifiable. While there is some evidence from rat and monkey studies that the corticospinal tract is relevant to mediate spinal motor plasticity (Wolpaw \& Lee, 1989; Chen \& Wolpaw, 2002), this has not been addressed systematically in humans.

Human studies are heterogeneous concerning training types, durations, localizations and assessment techniques (Voigt et al., 1998; Aagaard et al., 2002b; Carroll et al., 2002; Perez et al., 2004, 2006; Jensen et al., 2005; for review, see Enoka, 1997). Converse effects were observed on cortical and spinal plasticity, if tested after short-term (minutes to hours; Perez et al., 2004) and long-term (days to weeks) training (Taube et al., 2007). Interestingly, a general finding was the interdependence of training and task (Voigt et al., 1998; Jensen et al., 2005; Beck et al., 2007).

The present study was aimed at identifying spinal and supraspinal long-term effects of human lower-limb muscle training. While the H-reflex in soleus muscle (SOL) muscle was tested to detect spinal changes, a collision technique of subthreshold transcranial magnetic stimulation (TMS) and H-Reflex (Nielsen et al., 1993a; Petersen et al., 2003) was used to specifically assess the fast component of corticospinal projections. It was hypothesized that this pathway, comprising direct projections from the primary motor cortex, is affected by long-term training. Furthermore, training effects were expected to be observable in the active state and to be specific for the trained task. Therefore, two common training interventions for leg 
muscles enhancing explosive muscle strength [sensorimotor training (SMT) and ballistic strength training (BST)] were compared to a control group. Neurophysiological assessment was performed during two tasks which were, or were not, similar to one type of training. Hence, both training groups were tested in a trained and in a comparable nontrained task.

\section{Materials and methods Participants}

A total of 37 subjects participated in this study. They had no history of injuries of the ankle joint, seizures, neurosurgery or metal or electronic implants in their skull. All participants gave written informed consent to the experiments, which were approved by the local ethics committee of the University of Freiburg, and were in accordance with the Declaration of Helsinki. Participants did not perform any extra training while enrolled in the study and were randomly allocated to one of three treatment groups: SMT, BST and controls (CON); see Table 1.

\section{Training protocols}

Measurements were taken before and after a 4-week training period comprising 16 sessions of SMT or BST. All sessions were documented and supervised by the authors of the study. Training was performed according to a detailed schedule. One training session lasted from 45 to $55 \mathrm{~min}$ including $10 \mathrm{~min}$ warm-up and $10 \mathrm{~min}$ cool-down on a bicycle dynamometer at $100 \mathrm{~W}$. The pure training time amounted to $16 \mathrm{~min}$ with $9 \mathrm{~min}$ rest for the first four sessions and $24 \mathrm{~min}$ and $9 \mathrm{~min}$, respectively, for the 5 th -16 th sessions.

SMT consisted of a circle of postural stabilization tasks on four different devices: wobbling board, spinning top (Fig. 1a), soft mat (Airex ${ }^{\mathrm{TM}}$; Aalen, Germany) and cushion. Each type of exercise was performed on the right leg. with slightly flexed knee in an upright position looking straight forward and hands akimbo. The difficulty for SMT was further increased by performance of the training with eyes closed from the 12th to the 16th session (for the detailed training protocol see Gruber \& Gollhofer, 2004; Gruber et al., 2007a,b).

BST was performed according to protocols which have been used previously (Duchateau \& Hainaut, 1984; Van Cutsem et al., 1998). In a sitting position, subjects were instructed to start contractions with the ankle joint at $\sim 100^{\circ}$. Each training session consisted of four sets of 10 dorsal flexions and plantar extensions of the right ankle, respectively (Fig. 1b). The contractions were performed against loads of $30-40 \%$. of the individual one-repetition maximum. Loads were adjusted before the first training session and after 2 weeks. Between the contractions, subjects were instructed to rest for $2 \mathrm{~s}$ then concentrate on the next command to contract as fast as possible. Between the sets, there was a 3-5 min rest in order to avoid fatigue. The training was intensified by increasing the number of sets to six after four sessions, in line with the SMT group. The overall training time was matched in the two training groups. Both training regimes are known to enhance the maximum rate of force development $\left(\mathrm{RFD}_{\max }\right)$ rather than the maximum force (Gruber \& Gollhofer, 2004; Gruber et al., 2007a). The control group did not perform any particular training throughout the experimental period.

\section{Motor tasks}

The experiments were performed in two different motor tasks which were designed to match the training conditions. (i) Corresponding to BST, a dynamic dorsoflexing torque was applied at the ankle joints of both feet, which were placed on a motor-driven foot plate. Subjects were seated on a chair and instructed to counteract this torque as accurately and as soon as they were able to detect it. Peak torque was adjusted to the equivalent of $30-40 \%$ of the individual maximum torque. Dynamic torque pulses imposed on the subjects' ankle were controlled by a personal computer. Pulses were programmed with a time course of a sinusoidal profile and duration of $500 \mathrm{~ms}$ at random intervals. Between trials, subjects returned to the starting position. This position was adjusted individually before the testing to allow complete muscle relaxation due to the friction of the foot plate. The position of the right foot was continuously indicated to the subjects by an LED bar scale. Subjects were encouraged to react as fast and as precisely as possible when performing the compensatory plantar flexion (PFL) in order to keep the starting position stable on the LED scale and, thus, apply reproducible ankle torque. Movement of the foot plate was recorded by a goniometer and torque was measured by a torque meter. Baseline measurements without any stimulation were obtained together with background EMG from the right lower-leg muscles prior to and following the training. In order to test for functional training effects, torque measurements were further processed by differentiation to obtain $\mathrm{RFD}_{\text {nax }}$ as this parameter is known to be affected by both training regimes (Gruber \& Gollhofer, 2004; Gruber et al., 2007a,b). RFD ${ }_{\max }$ is an established parameter for testing the effects of ballistic ('explosive')-type strength training (Hakkinen \& Komi, 1983; Aagaard et al., 2002a). Differences in RFD max $_{\text {(post- }}$ minus pre-) were plotted over the relative changes in timing of $R F D_{\text {max }}$ during PFL (see Fig. 6). (ii) Corresponding to SMT, backward stance perturbation (PER) was used as a test for postural control. While standing on a computer-driven treadmill (Horstmann et al., 1987), a backward translation of the feet was induced. The amplitude was adjusted between 13 and $15 \mathrm{~cm}$ during pre-tests in order to match EMG for PFL and PER. The peak acceleration was $2.8 \mathrm{~m} / \mathrm{s}^{2}$ and the duration of the sinusoidal backward translation was $500 \mathrm{~ms}$, corresponding to the dorsoflexion torque applied in the sitting position. An upright starting position with minimal preactivation of the lower-leg muscles was maintained by means of visual feedback of the centre of pressure

TABLE 1. Biometric data of participating subjects

\begin{tabular}{lllllll}
\hline & \multicolumn{2}{l}{ Numbers of subjects } & & & \\
\cline { 2 - 6 } Training & Total & Male & All experiments* & Age (years) & Weight (kg) & Height (m) \\
\hline Sensory-motor training & 14 & 8 & $(8)$ & $26 \pm 3$ & $65 \pm 10$ & $1.73 \pm 0.1$ \\
Ballistic strength training & 12 & 7 & $(6)$ & $27 \pm 6$ & $67 \pm 14$ & $1.70 \pm 0.1$ \\
Controls & 11 & 7 & $(10)$ & $28 \pm 5$ & $67 \pm 7$ & $1.76 \pm 0.1$ \\
\hline
\end{tabular}

*Numbers in parentheses refer to subjects who participated in all experiments, i.e. those with sufficiently large H-reflex amplitudes which could be conditioned with TMS. 

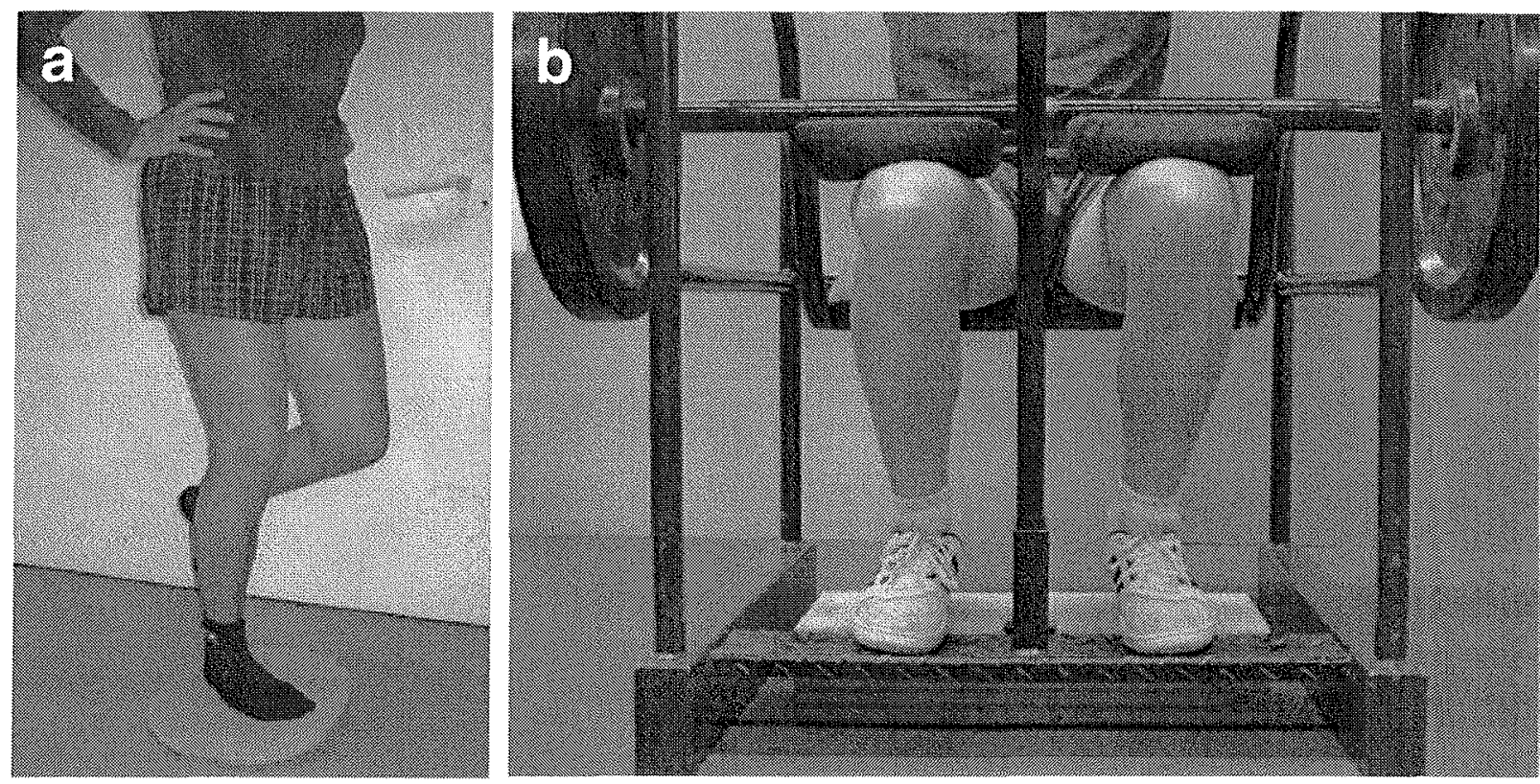

FIG. 1. Experimental setup illustrating the two types of training: (a), sensorimotor training, SMT; (b), ballistic strength training, BST.

on an LED bar. The subjects were instructed to maintain an upright position, compensating for the translatory perturbation and resuming their position as quickly as possible. After each perturbation, the belt was slowly moved back to the starting position. During perturbation, ankle joint movements were measured by goniometers fixed to the lateral side of the right foot and ground reaction forces were measured by force transducers underneath the treadmill.

\section{EMG}

EMG activity was measured with $\mathrm{Ag}-\mathrm{AgCl}$ surface cup-electrodes $(9 \mathrm{~mm}$ in diameter, centre-to-centre distance $2.5 \mathrm{~cm}$ ) filled with electrode jelly firmly attached to the skin over SOL, gastrocnemius medialis [GM; to double-check stability of maximum $M$-wave $\left(\mathrm{M}_{\text {max }}\right)$ during $\mathrm{H}$-reflex stimulation and to test for muscle-selective effects] and tibialis anterior (TA) muscles of the right leg. The longitudinal axis of the electrodes was in line with the presumed direction of the underlying muscle fibres. Electrode positions were carefully determined to ensure identical pre- and post- training recording sites. Interelectrode resistance was kept below $5 \mathrm{k} \Omega$ by means of shaving, light abrasion and degreasing of the skin. EMG signals were sampled at $4 \mathrm{kHz}$, amplified (gain $\times 1000)$ and band-pass filtered $(30-2000 \mathrm{~Hz}$ ). Root-mean-square values of the EMG from single trials were calculated as mean average voltage of $100-\mathrm{ms}$ intervals starting from EMG onset after PER or PFL. SOL EMG was expressed as a percentage of SOL $\mathrm{M}_{\max }$. Onset of EMG was determined as the moment when EMG exceeded resting baseline values by $2 \mathrm{SD}$.

\section{H-reflex}

SOL H-reflexes were evoked with a 0.5 -ms current pulse over the tibial nerve in the popliteal fossa with a constant current square wave stimulator (Digitimer DS7). A $10 \times 5 \mathrm{~cm}$ graphite rubber anode was placed below the patella and a $2 \mathrm{~cm}$ diameter electrode was used as a cathode. Electrode positioning was done in stance and checked in the sitting position before the experiments were conducted. The sizes of the H-refiex and of the M-response were measured as peak-to-peakamplitudes. A constant interstimulus interval (ISI) of $3 \mathrm{~s}$ was used between successive electric stimuli. The current was progressively increased until $M_{\max }$ was obtained. $M_{\max }$ was verified and maximum H-reflex amplitude $\left(\mathrm{H}_{\max }\right)$ was stored for calculation of $\mathrm{H}_{\max } / \mathrm{M}_{\max }$ ratio. H-reflex size and $M_{\max }$ were checked 6-8 times per experiment to ensure reproducibility and appropriate stimulation intensity, particularly in the H-reflex-conditioning experiment (see below). H-reflex testing was performed during a dynamic PFL matching BST and in the rest condition, sitting position (REST). Corresponding to SMT, $\mathrm{H}$-reflex testing was also performed during PER and, for comparison with the REST condition, during maintenance of a quiet standing position stance (STANCE).

\section{H-reflex conditioning with TMS}

TMS was applied with a commercially available stimulator (MagPro200'M ; Magstim Company, Dyfed, UK; maximum magnetic field strength 2 Tesla). The stimulus waveform was monophasic with a pulse width of $200 \mu \mathrm{s}$. A circular coil (diameter $90 \mathrm{~mm}$ ) was used, current flowing clockwise. Motor evoked potentials (MEP) in the TA and SOL were evoked by TMS of the contralateral motor cortical leg area. At the beginning of each experiment the coil position was systematically changed in order to determine the hot-spot for the TA. This muscle was selected because its MEP is easily evoked at rest compared to SOL. Usually, placement of the coil was started slightly anterior and $0-2 \mathrm{~cm}$ lateral to $\mathrm{Cz}$ (according to the international $10-20$ system of EEG electrode placement) and then optimized in order to produce MEP with the lowest stimulus strength. This position was marked on the scalp and controlled during stimulation. The subject was in a reclined position placing his head in a solid concave head rest that was attached to the chair. The head of the subject was held against the head rest using a rubber band. The coil, by means of a coil holder, was also attached to the chair. During perturbation, subjects wore a 
harness with a dorsal head rest and a clamping device to hold the coil (Schubert et al., 1997). While performing the experiments in the sitting position and during stance, the coil position was checked four or five times during each experiment with reference to the position marked on the scalp. Control stimuli assured that the placement was stable.

Resting motor threshold (MT) for TA and SOL were determined in steps of $1 \%$ of the maximum stimulator output. MT was defined as the minimal stimulus intensity required for inducing MEP amplitudes of $>100 \mu \mathrm{V}$ in at least three of five consecutive trials. Stimulation strength and stability of MT was determined in TA as this was always lower than MT in SOL. After determination of MT, TMS stimulation strength was adjusted for SOL H-reflex conditioning at subthreshold intensity $(\sim 0.7 \times$ resting MT of TA). In order to keep TMS intensity below threshold, the EMG was continuously screened for MEPs in TA or SOL in single trials and for the maximum amplitude of SOL EMG following TMS in the relevant time interval being $<5 \%$ of $\mathrm{M}_{\max }$ in an average of 10 trials.

It was demonstrated that the susceptibility of the H-reflex to conditioning depends on the size of the control reflex (Crone et al., 1990). It may be assumed that it reflects spinal motoneuron excitability on a largely linear scale, if the test reflex is adjusted to a size of $15-25 \% \mathrm{M}_{\max }$ and if this control H-reflex size is tested on the ascending part of the $\mathrm{H}-\mathrm{M}$ recruitment curve. In the present experiments, this was tested by means of $\mathrm{H}-\mathrm{M}$ recruitment curves recorded prior to and after the SOL H-reflex conditioning experiment (compare small inlays in Fig. 4). In two subjects (one in each training group) with small H-reflexes we used $33 \%$ of $\mathrm{H}_{\max }$ as control size of the H-reflex. SOL conditioned H-reflex $\left(\mathrm{H}_{\text {cond }}\right)$ with subthreshold TMS was performed according to the protocol established by Nielsen and coworkers (Nielsen et al., 1993b; Nielsen \& Petersen, 1995; Petersen et al., 1998). TMS and electric tibial nerve stimulation for $\mathrm{H}$-reflex testing were applied in a random order at various ISIs. ISI were assessed from -8 to $0 \mathrm{~ms}$ (in steps of $1 \mathrm{~ms}$ ) and at $3,6,9,12$ and $15 \mathrm{~ms}$, expressed as the time interval between electrical and magnetic stimulation. This time course was used to determine the first $0.5-1 \mathrm{~ms}$ of TMS-induced H-reffex facilitation to specifically assess the fast component of corticospinal projections (termed short-latency facilitation of $\mathrm{H}_{\text {cond }}$ ). Negative intervals indicate that the electric pulse evoking the test $\mathrm{H}$-reflex preceded the conditioning magnetic pulse. Ten stimuli were recorded for each ISI and for two control conditions with either single peripheral nerve stimulation or with TMS only. All TMS experiments were performed at a random interval of at least $5 \mathrm{~s}$ between stimulations. The intensity of the magnetic stimulus was adjusted at the beginning of each experiment to be below the threshold for eliciting a direct MEP in the TA and SOL even during strong PFL. A time course of ISIs to assess the effect of the magnetic stimulus on the SOL H-reflex was first obtained, while the subject performed dynamic PFL. ISI time course was then confirmed while performing a tonic PFL and then tested during rest. If the magnetic stimulus had an effect on the H-reflex while the subject was at rest, the intensity of stimulation was decreased further until no effects were observed. If the short-latency facilitation of $\mathrm{H}_{\text {cond }}$ could be reproduced in tonic and dynamic PFL, reproducibility of the time course was assumed. Due to time limits in the experiment itself in order to avoid fatigue, only ISIs appropriate for eliciting short-latency facilitation of $\mathrm{H}_{\text {cond }}$ together with one preceding and one subsequent ISI in the time slot for shortlatency facilitation and the first one or two ISIs with inhibition of $\mathrm{H}_{\text {cond }}$ in the ISI time slot for late effects (ISI 6, 9, 12 and $15 \mathrm{~ms}$ ) were tested in the PER condition and when the measurements were repeated after the training. Reproducibility in the standing position had been confirmed in preliminary experiments and reproducibility in the PER condition was also confirmed in six subjects from each training group by testing additional ISIs prior to the determined onset of early facilitation.

\section{General experimental procedure}

The experiments were conducted before and after the training period. The post-training experiment took place $24-72 \mathrm{~h}$ after the last training session in order to avoid fatigue. For both active tasks (PFL and PER), experiments were conducted in the same order in all subjects. To become acquainted, subjects first performed each task 10-20 times without any stimulation until producing stable responses in the EMG signal. Then, 10 trials were recorded and EMG was rectified and averaged. The latency for the first peak of EMG activity was individually determined. Four different torque amplitude factors $(24,32,40$ and $48 \%$ of maximum torque) were tested in PFL and amplitude of PER was varied between 13 and $15 \mathrm{~cm}$ in order to attain equivalent slopes and levels of SOL EMG activity during the first EMG peak in both motor tasks. An example of a single subject's recording after this matching procedure is shown in Fig. 2A for PER and in Fig. 2B for PFL. After such individual matching of the EMG in both active tasks, the same combination of mechanical stimulation conditions during PFL and PER (torque amplitude and perturbation amplitude) was always used for testing prior to and after training in this individual.

In order to standardize H-reflex testing during the dynamic tasks (PER and PFL), responses were timed to coincide with the first peak of SOL EMG (compare traces at grey bar, Fig. 2) which was induced by the mechanical stimulus of PER or when dorsoflexion torque was imposed during PFL. In the same way, $\mathrm{H}$-reflexes conditioned with TMS (see below) were also timed to coincide with the first SOL EMG peak. As PER and PFL were previously adjusted to produce similar EMG levels during the initial EMG peak, stimulation always coincided with similar levels of background EMG. Peripheral nerve stimulation was applied during movement and at rest to evaluate spinal excitability. Corticospinal excitability was tested during rest and active tasks using subthreshold TMS to condition the SOL H-reflex. According to Nielsen and coworkers, an assessment of functional activation within direct corticospinal pathways is possible by this H-reflex conditioning with subthreshold TMS, because short-latency H-reflex facilitation will reflect functional activation of the pathway if stimulation with TMS is subliminal and both peripheral nerve and TMS are kept constant throughout the experiment (Nielsen et al., 1993b; Nielsen \& Petersen, 1995; Petersen et al., 1998). Thus, each subject was tested in three experiments. (i) PFL and PER alone to match EMG and determine functional training effects from PFL task after training. (ii) PFL and PER plus electrical stimulation (SOL H-reflexes) to assess spinal excitability at rest and during functional activation by PFL and PER. (iii) PFL and PER plus SOL H-reflex (test reflex) plus subthreshold TMS (conditioning stimulus) to assess the contribution of fast (possibly monosynaptic) corticospinal pathways to functional EMG responses in PFL and PER.

\section{Statistical analysis}

Significant short-latency facilitation was confirmed by $t$-test of differences between conditioned and test H-reflex. Group data are presented as mean values \pm SEM. To test for significant training effects, an ANOVA was applied with the factors training group and task, the latter being calculated as the difference between data obtained 


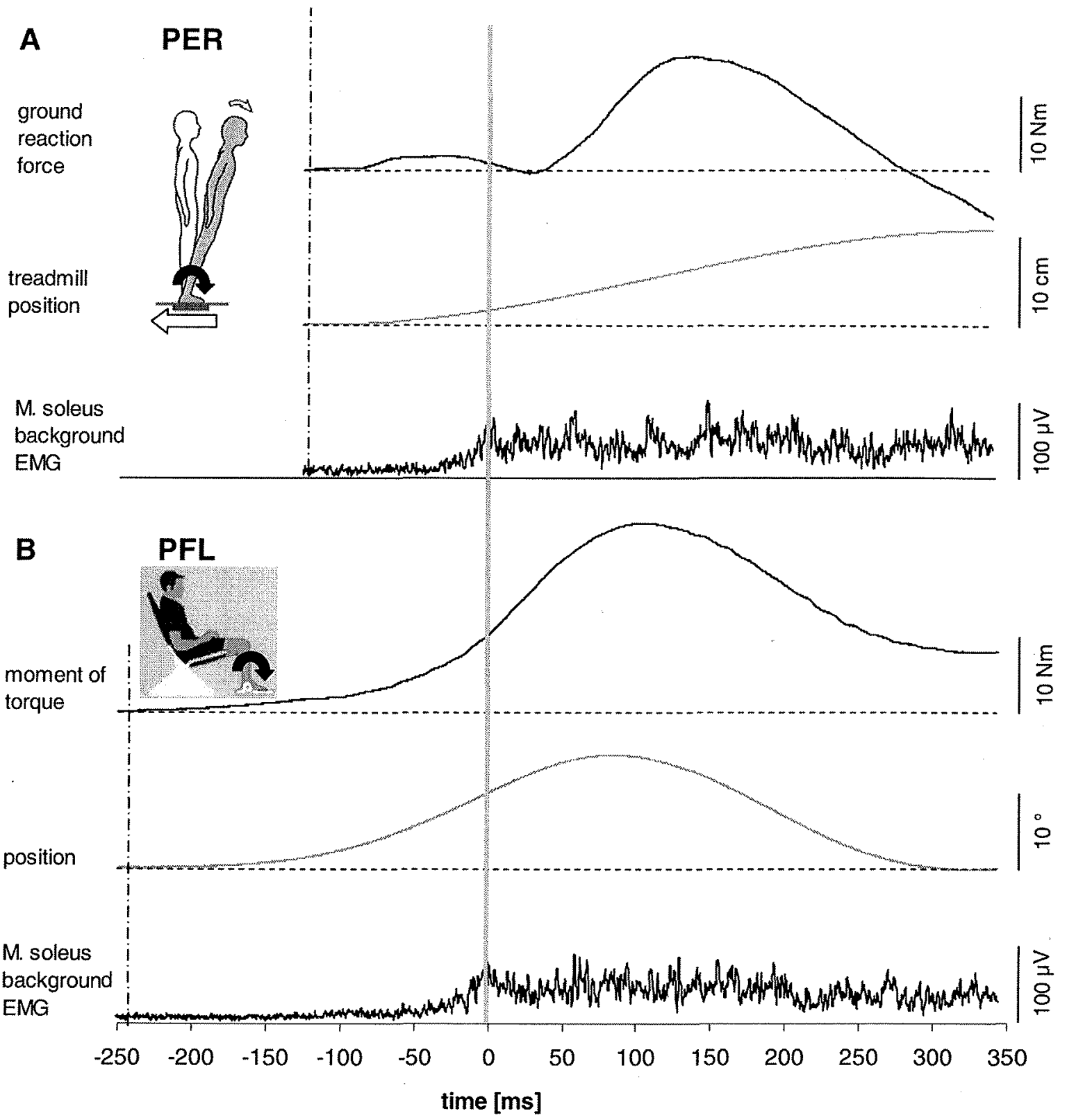

FIG. 2. Motor tasks matching the training conditions: original single-subject recording of (A) PER and (B) PFL; see insets for illustration of the motor task. Traces show means of $n=10$ trials; EMG was rectified before summation. The broken line indicates the beginning of mechanical perturbation in both tasks; recordings were aligned with respect to EMG onset. The thick grey line indicates the moment when the H-reflex was timed to occur in the following trials during electrophysiological testing.

prior to training and that measured after training (repeated-measurements design, level of significance $P<0.05$ ). To evaluate significant before-after differences in each training group, a one-factorial ANOVA was applied to test the null hypothesis that differences between measurements taken prior to and following the training were equal to zero. In addition, Wilcoxon signed-rank tests for paired samples (twotailed, level of significance $P<0.05$ ) were calculated where group size was $<10$ (which applied to the results obtained from the experiment with TMS conditioning of the H-reflex due to drop-outs with insufficient H-reflex size). All analyses were executed using SPSS 11.5 software.

\section{Results}

\section{H-reflex conditioning by subthreshold TMS}

H-reflexes were conditioned with subthreshold TMS in order to assess ongoing activation of corticospinal drive during movement and to compare this drive prior to and after training. A short-latency facilitation was found in all tested subjects and in all active conditions for negative conditioning-test intervals when peripheral nerve stimulation preceded TMS (see Fig. 3). Its onset varied between -5 and $-2 \mathrm{~ms}$ in the different subjects. This difference in latency is most probably explained by anatomical conditions, namely 


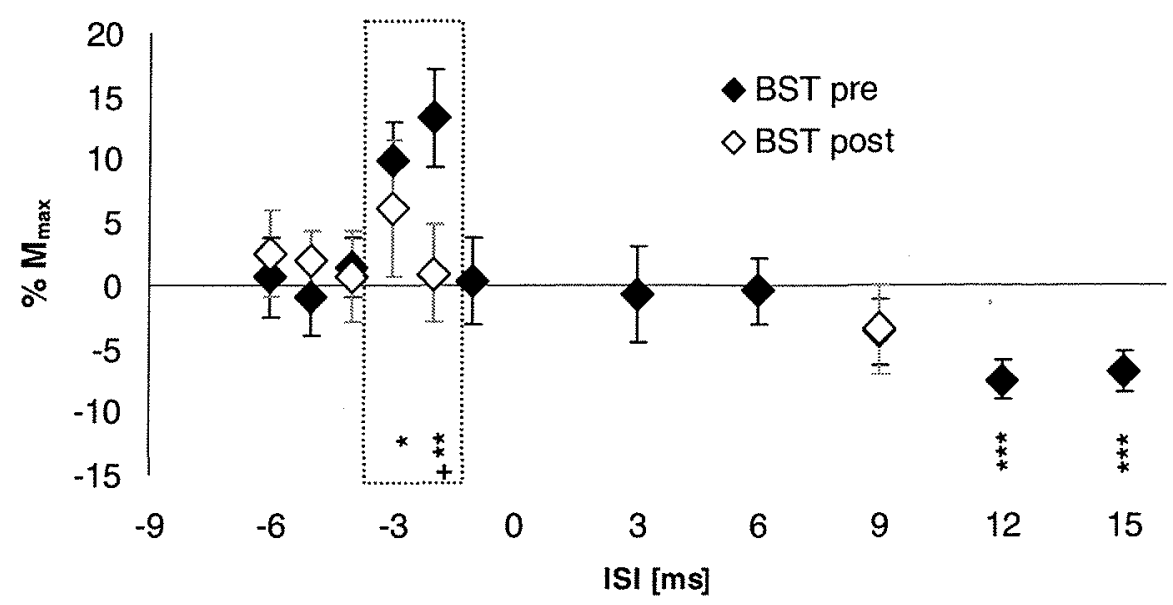

FIG. 3. Time-course of H-reflex conditioning with subthreshold TMS; ISIs (abscissa) $<0$ indicate that tibial nerve stimulation preceded TMS. Recordings were obtained during the PFL task from a single subject enrolled in the BST training group. Each dot indicates averages of 10 H-reflexes, expressed as the difference between $\mathrm{H}_{\text {cond }}$ and $\mathrm{H}_{\mathrm{ctrl}}$ as percentage of $\mathrm{M}_{\text {max }}$; SEMs are shown by the bars. $* P<0.05$, **P<0.01 and ***P<0.005 vs. zero; $P<0.05$, pre- vs. post-training trials. The dotted rectangle shows the ISIs at which short-latency facilitation occurred.

differences in the height of the subjects and in the conduction velocity of their central and/or peripheral fibres (Nielsen \& Petersen, 1995). Therefore, the lowest ISI which resulted in a significant shortlatency facilitation as derived from the PFL condition was used throughout the experiment after confirmation in the tonic condition for each subject. Reproducibility in the PER condition was confirmed in six subjects from each training group by testing additional ISIs prior to the determined onset of early facilitation. Positive ISIs $<+6 \mathrm{~ms}$ (ranging from +6 to $+9 \mathrm{~ms}$ between individuals) resulted either in a long-latency facilitation or inhibition; in line with results presented by Petersen et al. (1998), we observed a longlatency facilitation during tonic activation and stance (not shown) whereas an inhibitory effect was observed during the tasks with dynamic activation of SOL, i.e. PFL and PER. When plotting conditioned H-reflex size as a function of ISI between TMS and electrical stimulation, a typical time course of $\mathrm{H}$-reflex modulation was obtained with short-latency H-reflex facilitation and long-latency $\mathrm{H}$-reflex inhibition. An example of this ISI time course during PFL is shown in Fig. 3. H-reflex amplitudes for each ISI are expressed as percentage of $\mathrm{M}_{\max }$ according to the formula $\left(\mathrm{H}_{\text {cond }}-\mathrm{H}_{\mathrm{crr}}\right) / \mathrm{Mmax}$. A similar time course was observed in all 24 subjects in whom the conditioning of the H-reflex could be tested. A two-way ANOVA ( $n=24$ subjects from the pre-training recording) showed highly significant modulation of $\mathrm{H}_{\text {cond }}$ size for different ISIs $(P<0.0001)$ while this modulation was not different between training groups (n.s.). ISI time course in single subjects was well reproducible after training (Fig. 3) and then showed subtle changes for ISI with shortlatency H-reflex facilitation.

The short-latency H-reflex facilitation during functional SOL activation is supposed to be a correlate of its direct corticospinal activation (Nielsen et al., 1993b; Nielsen \& Petersen, 1995; Petersen et al., 1998, 2003). Therefore, the extent of this facilitation was considered relevant for the assessment of training effects in fast corrective ankle movements. H-reflex recordings with ISIs inducing short-latency H-reflex facilitation are shown for a single subject from each training group. A complete set of recordings with both tasks (PFL left, PER right; Fig. 4) tested before and after 4 weeks of training is shown for each subject.

Averages from 10 randomized trials for control (thick lines) and conditioned (thin lines) H-reflexes were superimposed to illustrate the conditioning effect of subliminal TMS. Amplitude changes in $\mathrm{H}_{\text {cond }}$ compared with control H-reflex were found to be modulated systematically for all tested subjects who had been enrolled in one of the training groups. Figure 5 shows normalized individual results for short-latency facilitation of $\mathrm{H}_{\text {cond. }}$. Short-latency $\mathrm{H}$-reflex facilitation ranged from 1 to $16 \%$ of $\mathrm{M}_{\max }$ (before training, $6.3 \pm 0.1 \%$ and $4.6 \pm 0.1 \%$ of $M_{\max }$ for PFL and PER, respectively; difference between PFL and PER n.s.). A comparison of measurements obtained prior to and after training (left and right columns, respectively) indicates differential effects resulting from an interaction of training with task (ANOVA training $\times$ task, $P<0.005$ ): during PFL (a task which was not specifically trained by SMT), SMT resulted in an increase while BST reduced early facilitation. Conversely, an increase in short-latency facilitation was observed in all but one BST subjects when they were challenged with PER (a task which was not specifically trained by BST). PER after SMT did not induce significant group effects but six out of eight SM-trained subjects showed decreased early facilitation. In order to check for muscleselective effects, the GM H-reflex was also evaluated in this task. A similar reduction was found for the conditioning effects in the GM H-reflex.

Grand mean changes in short-latency facilitation after training amounted to $3.3 \pm 0.3 \%$ of $\mathrm{M}_{\max }$ during PFL following SMT. After BST, changes of $-4.1 \pm 0.5 \%$ of $\mathrm{M}_{\max }$ and $4.7 \pm 0.5 \%$ of $\mathrm{M}_{\max }$ were observed during PFL and PER, respectively. No systematic effects were found in the control group (bottom diagrams).

\section{Rate of force development}

A significant training effect on $\mathrm{RFD}_{\max }$ was observed during PFL following BST only $(+70 \pm 44 \mathrm{Nm} / \mathrm{s}, P<0.01)$, while $\mathrm{RFD}_{\max }$ 
plantarflexion
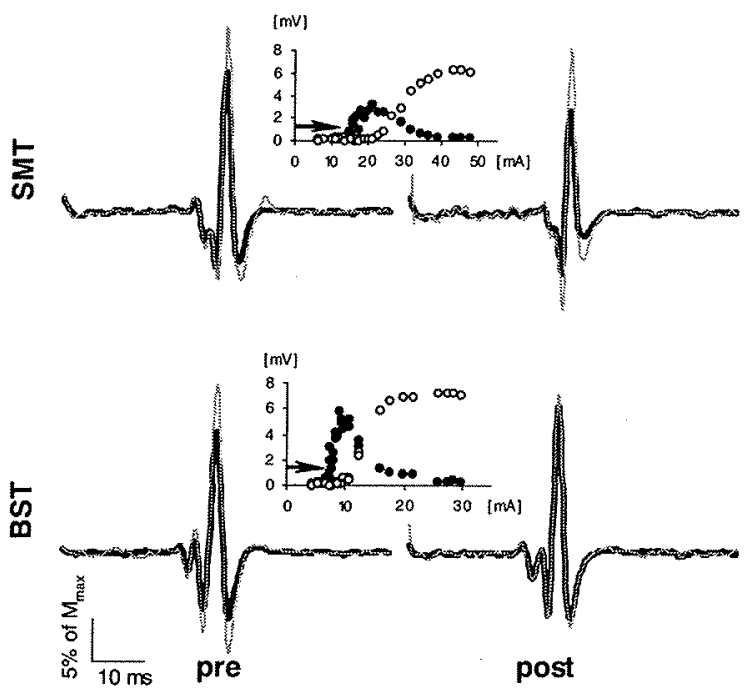

perturbation
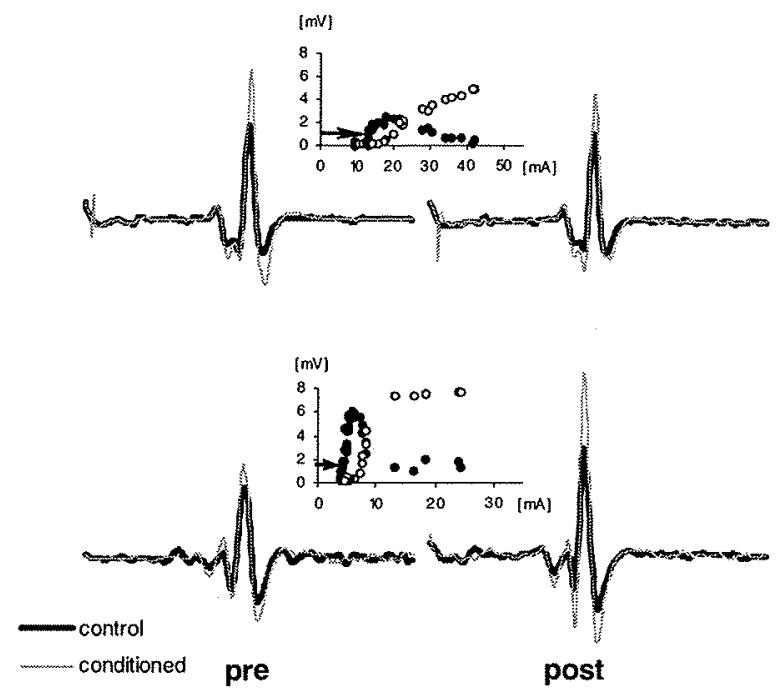

Fig. 4. Original recording of control (thick lines) and conditioned (thin grey lines) H-reflexes from a subject from each training group (SMT, top; BST, bottom) during both tasks (PFL, left group; PER, right group of figures). Insets indicate how peripheral nerve stimulation strength was adjusted to produce an H-reflex of $15-25 \%$ of individual $M_{\max }$ on the ascending slope of the H-reflex recruitment curve (open circles indicate M-response, filled dots represent $\mathrm{H}$-response and small arrows indicate the size of H-reflex used in the experiment). Each trace is the average of at least $10 \mathrm{H}$-reflex recordings conditioned with TMS at ISIs of $-4 \mathrm{~ms}$ for the subject enrolled in the SMT group and of $-2 \mathrm{~ms}$ for subject in the BST group (these were the individual ISIs with first appearance of short-latency facilitation in each subject). For normalization and interindividual comparison, H-reflex amplitude is expressed as a percentage of $M_{\max }$ which was obtained during the respective tasks (PFL or PER)

was shifted in time to occur significantly earlier in both training groups (BST, $-31 \pm 17 \mathrm{~ms}, \quad P<0.01$; SMT, $\quad-21 \pm 11 \mathrm{~ms}$, $P<0.01$ ). The difference between $\mathrm{RFD}_{\max }$ in BST and SMT almost reached significance $(P=0.07)$. $R F D_{\max }$ was also analysed for PER in the two training conditions. There were no significant training effects on $\mathrm{RFD}_{\max }$ (expressed as difference after minus before) in any of the training groups. There was no change in the control group.

\section{EMG}

Before the training, no difference was observed for the EMG between PFL and PER during the initial $100 \mathrm{~ms}$ of task-induced activity in SOL (mean average voltage expressed as percentage of $\mathrm{M}_{\max }$; see Materials and methods). This lack of EMG difference between PFL and PER indicated that matching of the tasks for similar EMG activity was successful. Comparison of SOL EMG before and after training revealed a significant training effect and interaction with training group (differences in normalized SOL EMG between the first and second assessment/after training for PFL and PER conditions were, for PFL: BST, $0.47 \pm 0.24 \%$ of $\mathrm{M}_{\max } ; \mathrm{SMT},-0.03 \pm 0.11 \%$ of $\mathrm{M}_{\max } ; \mathrm{CON},-0.09 \pm 0.12 \%$ of $\mathrm{M}_{\max }$; and for PER: BST, $0.05 \pm 0.13 \%$ of $\mathrm{M}_{\max }$; SMT, $0.29 \pm 0.13 \%$ of $\mathrm{M}_{\max } ; \quad \mathrm{CON}, \quad-0.07 \% \pm 0.15 \%$ of $\mathrm{M}_{\max }$; repeated-measures ANOVA, $P<0.05$ ). Post hoc analysis revealed increased SOL EMG after BST as compared to CON in the PFL condition (BST vs. CON and vs. SMT, $P<0.05$ ) while no changes were observed for SMT in the PER condition (SMT vs. BST, $P=0.12$ ). This may be seen as a direct effect of training which is in line with a significant $R_{F D}$ max increase in the BST group (see Fig. 6). EMG from other time intervals and from GM up to $500 \mathrm{~ms}$ after movement onset showed no significant differences irrespective of the type of training.

\section{H-reflex modulation}

$\mathrm{H}$-reflex amplitudes were expressed as percentages of maximum M-wave. $H_{\max } / \mathrm{M}_{\max }$ ratios were significantly modulated by motor tasks. The ratio was increased for PER compared to STANCE (61 \pm 3 vs. $48 \pm 4 \%, P<0.01)$. PFL compared to REST showed a trend to increase (63 \pm 3 vs. $55 \pm 4 \%, P=0.087)$ and $\mathrm{PFL}$ compared to STANCE was clearly increased ( $63 \pm 3$ vs. $48 \pm 4 \% ; P<0.005)$. This pattern of task-related H-reflex modulation was similar in all three groups (before training: ANOVA repeated-measures design, interaction task $\times$ treatment, n.s.) and was not affected by training (after training: ANOVA interaction task $\times$ treatment $\times$ time, n.s.). This was also true when individually and task-wise subtracting pretraining $\mathrm{H}_{\max } / \mathrm{M}_{\max }$ ratios from post-training results. The differences between these $\mathrm{H}_{\max } / \mathrm{M}_{\max }$ ratios were not systematically affected irrespective of whether the active conditions (PFL, PER) or corresponding resting conditions (REST, STANCE) were compared between groups.

\section{Discussion}

Fast corticospinal projections were systematically modulated by strength and balance training. Spinal excitability, as assessed by SOL H-reflex testing, was unaltered after 4 weeks of training, indicating that supraspinal sites are essential for long-term plasticity. Modulation of corticospinal excitability was observed to be interdependent with training and with motor task. The short-latency facilitation of conditioned $\mathrm{H}$-reflex was diminished in the trained task 
PFL
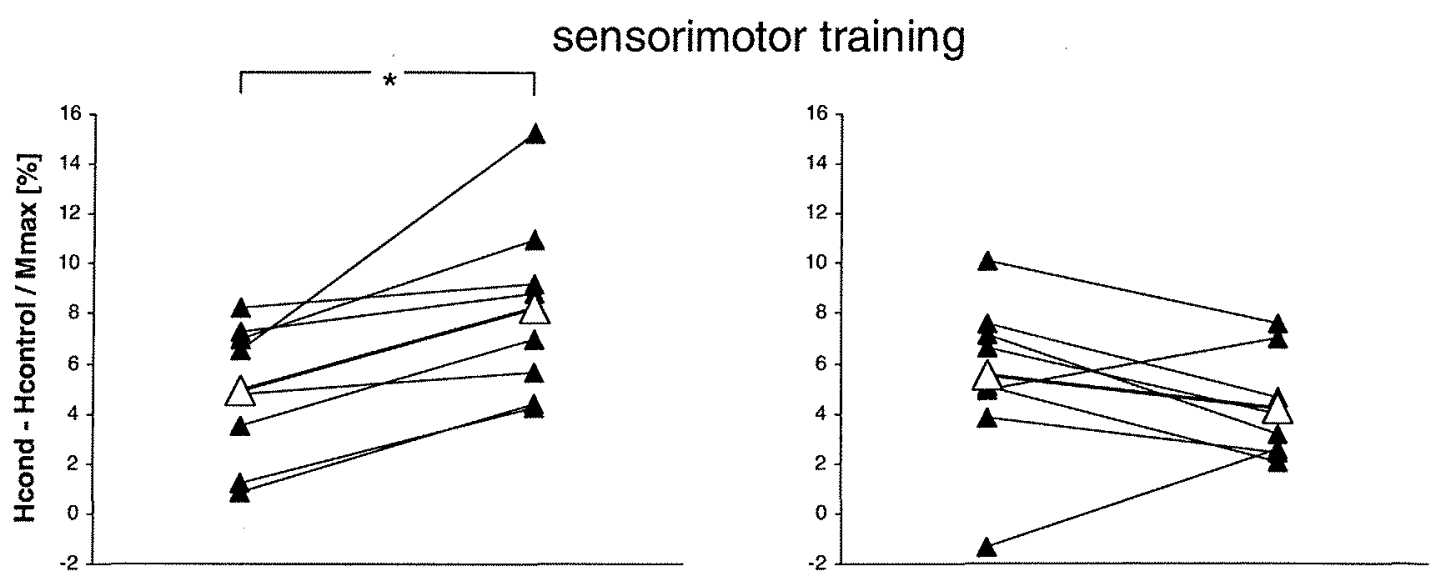

ballistic strength training
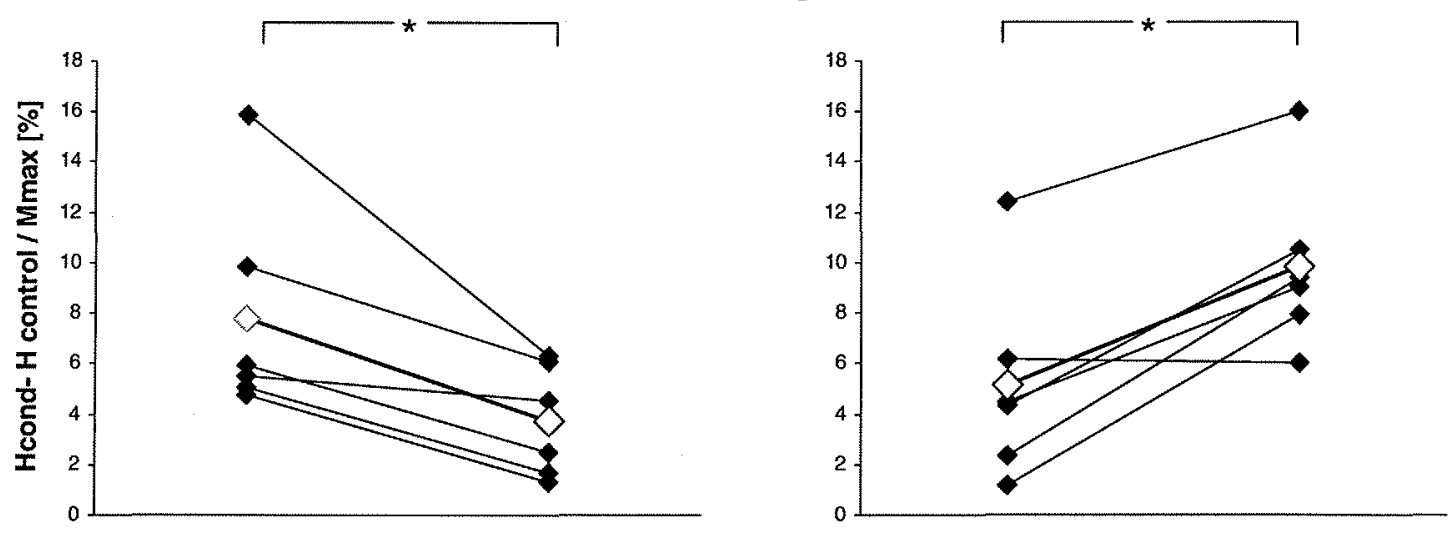

\section{control}
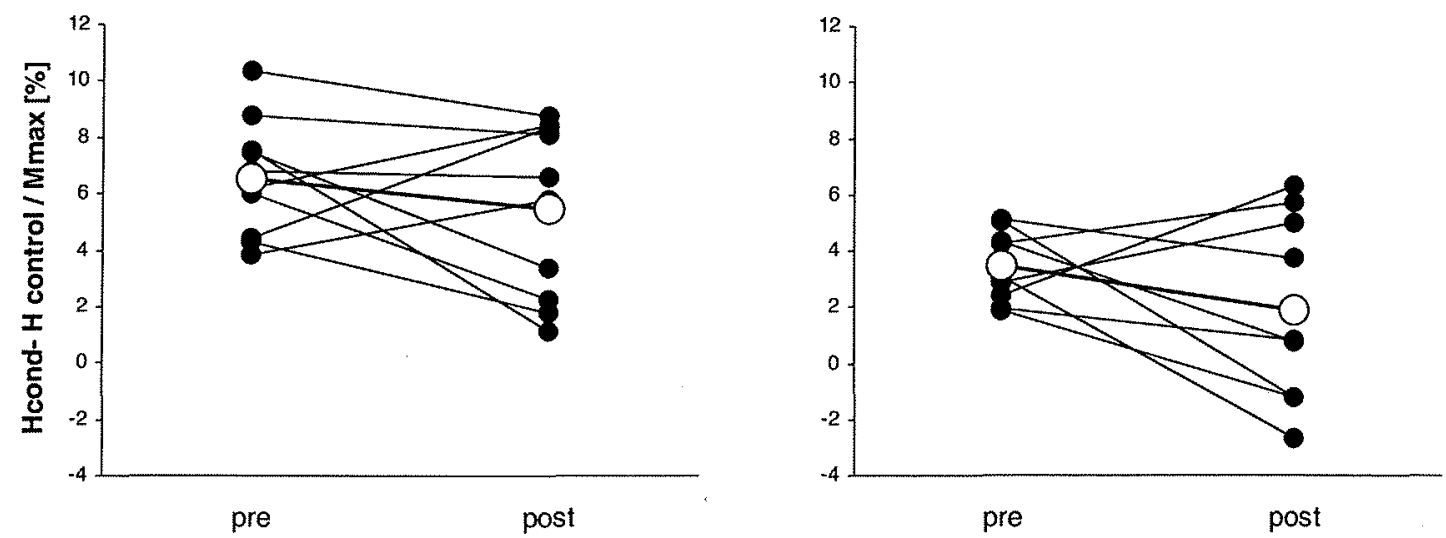

FIG. 5. Effect of training on short-latency facilitation during PFL (sitting condition, left) and PER (standing on treadmill, right). The ISI for which the first significant facilitation was observed, ranging from -5 to $-2 \mathrm{~ms}$ in different individuals, was compared before and after the intervention. ${ }^{*} P<0.05$ for $\mathrm{H}-$ reflex facilitation prior to vs. following training (Wilcoxon signed-rank test).

and increased in the nontrained task. Furthermore, neural plasticity was observable in a functional state only. On a functional level it was shown that both types of training increased the rate of force development, suggesting that corticospinal projections play a role in training-induced adaptation of leg motor control in humans. This is noteworthy, as direct corticospinal projections to the lower limb 


\section{PFL}

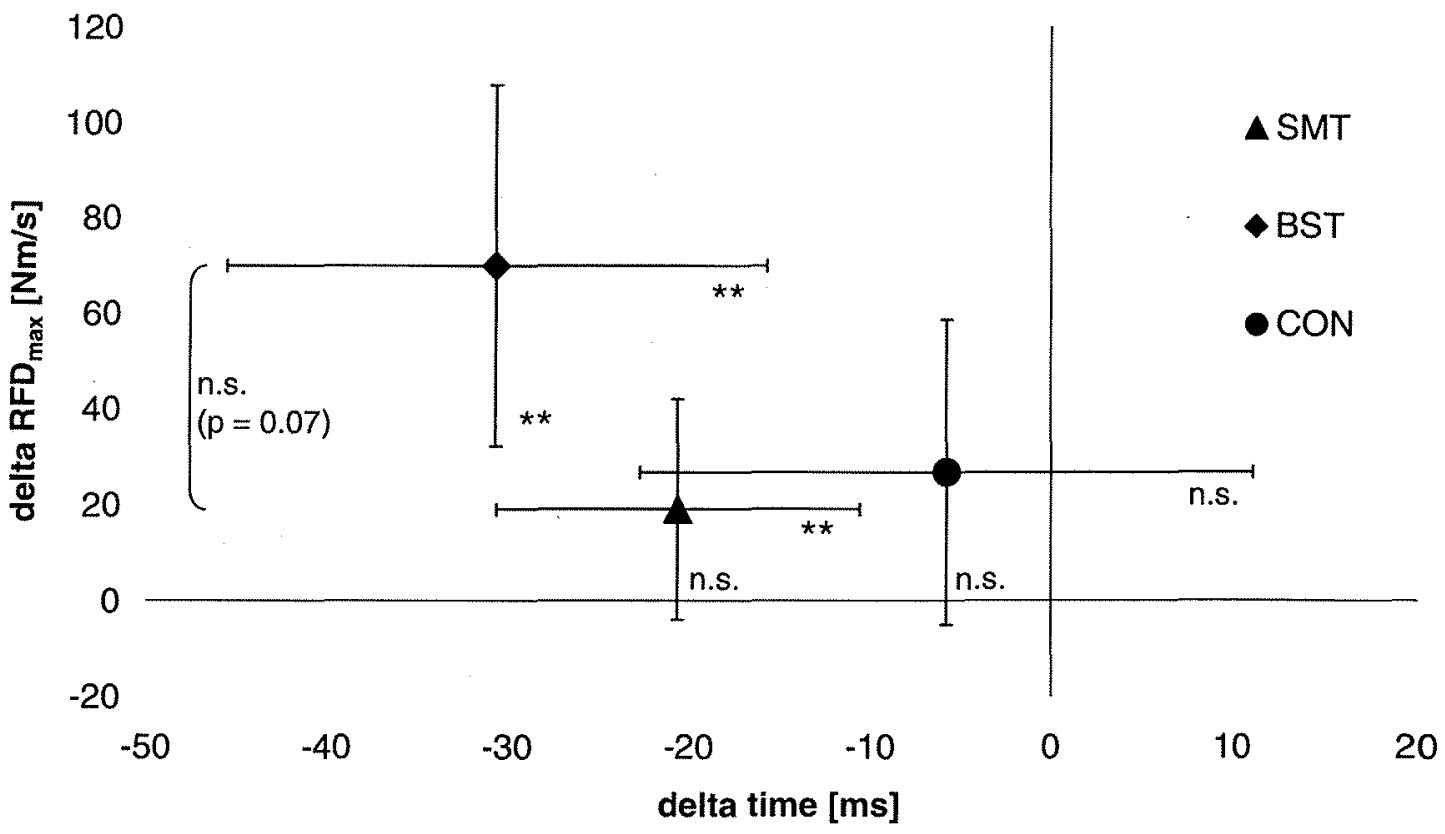

F1G. 6. This graph shows how training affected the maximum rate of force development and how its occurrence shifted. The average of all single subjects' RFD ${ }_{\text {max }}$ ( $\triangle \mathrm{RFD}$, derivative of torque, the difference between measurements prior to training and those after training; $y$-axis) was plotted over the corresponding time shift of RFD occurrence (before training to after training; $x$-axis) during PFL. Grand means for controls (O): CON, $n=9$; sensorimotor trained ( $\Delta$ ) SMT, $n=13$; and ballistic strength trained $(\bullet) \mathrm{BST}, n=10$ subjects; error bars indicate $\pm 95 \%$ confidence interval of the mean. Significance levels: $* * P<0.01$; n.s., not significant (one-sample analysis, $t$-test, hypothesized mean $=0$ ); bracket indicates trend for group difference (Scheffé, post hoc).

muscles are probably unique to high primates and humans (Jankowska et al., 1975).

\section{A case for corticospinal training effects}

As corticospinal excitability was assessed during a functional task, it was necessary to keep spinal motoneuron excitability at a constant level. This was achieved by adjusting the control $\mathrm{H}$-reflex to a target value of $20 \%$ of $\mathrm{M}_{\max }(15-25 \%$ was accepted) in measurements before and after training (Crone et al., 1990; Nielsen et al., 1993b; Taube et al., 2007). The so-called conditioning of this standardized test H-reflex by a collision with subthreshold TMS produces a precise pattern of facilitation and inhibition when the ISI between test and conditioning stimulus is varied. The high temporal resolution of this technique allows investigation of specific components of the corticospinal volley (Nielsen et al., 1993b). Short-latency, presumably monosynaptic, projections may thereby be distinguished from polysynaptic pathways with longer latencies (Petersen et al., 1998, 2003). The early part of the subthreshold conditioning stimulus most probaby activates direct monosynaptic projections and is not influenced by presynaptic inhibition provided that the ISI used is short enough to exclude nonmonosynaptic effects. These projections are also considered unaffected by presynaptic inhibition in humans (Nielsen \& Petersen, 1994). Therefore, modulation of $\mathrm{H}_{\text {cond }}$ facilitation following training may point either towards changes in cortical excitability or to changes in the firing rate of the spinal motoneurons or their intrinsic firing properties. However, adaptations of the latter should have become obvious in terms of the $\mathrm{H}_{\max } / \mathrm{M}_{\max }$ ratio, which was not modulated by training. Hence, changes in cortical motoneuron excitability were most probably responsible for the modulation of $\mathrm{H}_{\text {cond }}$. Accordingly, significant modulation of the earliest observable facilitation, the so-called short-latency facilitation of $\mathrm{H}_{\text {cond }}$, contributes to the notion of training-related modulation of fast-conducting, possibly direct, corticospinal fibres to SOL.

\section{How can a simultaneous decrease and increase in excitability be explained?}

After training, we observed converse effects during the trained task and during a similar, but nontrained, motor task. The results show that corticospinal excitability was increased or decreased following training but this was not a general effect as it could not be detected at rest. The interaction effect of task with training suggests that changes in $\mathrm{H}_{\text {cond }}$ facilitation were related to the particular match of task-dependent activation and corresponding training. The lack of effects in the control group indicates that changes in $\mathrm{H}_{\text {cond }}$ facilitation were related to training. It can be assumed that modulation of $H_{\text {cond }}$ is not merely a function of task-related activation, but facilitation was reduced or increased depending upon whether a task had been practised or not (Fig. 5). Most previous training studies have shown only unidirectional cortical and corticospinal adaptations after lowerleg force and balance training. A modulation of the activity within corticospinal projections was observed after several weeks of training and this was a common finding of several former long-term training studies in the upper and lower limb, although the motor conditions under which they were observed were not uniform (Carroll et al., 
2002; Jensen et al., 2005; Beck et al., 2007; Beck, 2007; Taube et al., 2007). In particular, the effect of varying motor tasks had not been tested before. When this was first attempted in our earlier study applying the same methodology, results showed high task and training specificity and training effects were only observable during performance of the trained task (Beck et al., 2007). For the anterior tibial muscle, Perez et al., 2004) demonstrated that $32 \mathrm{~min}$ of motor skill training increased MEP recruitment and maximal MEP amplitudes. In a subsequent study using the same training the authors observed a significant increase in EEG-EMG coherence, suggesting that modulation of corticospinal drive takes part in the motor skill leaming process (Perez et al., 2006). For unstable stance it was shown that the long-latency reflex of the SOL muscle is under supraspinal control, while short- and medium-latency responses are not (Taube et al., 2006). In a subsequent study, the affects of long-term balance training (SMT) on the spinal short-latency reflex and on the transcortical longlatency reflex loop were compared and the results showed a reduction in corticospinal excitability after training at the long-latency reflex latency only (Taube et al., 2007). This reduction was inversely related to improvements in balance performance, suggesting that it is mainly due to supraspinal training adaptations.

The present results are well in line with the notion of corticospinal plasticity following exercise of fast voluntary leg motor control. They further extend the former findings by demonstrating that traininginduced modulation of fast corticospinal connections is rapidly adaptable and task-dependent. Effects of these projections can be reversed just by changing the motor task from the trained to a nontrained condition with similar motor characteristics.

\section{Task specificity of training effects: study limitations}

Significant effects of training on $\mathrm{RFD}_{\max }$ were only observed when testing the trained condition (sitting, PFL) but not in the postural task. This would be well in line with training specificity as training effects are known to be confined to the practiced task (Voigt et al., 1998; Beck et al., 2007; Taube et al., 2007). Accordingly, perturbation of stance at a low acceleration does not require maximum RFD and may therefore not have affected $\mathrm{RFD}_{\max }$ during perturbed stance after any training. Much in line with this it must be considered that musclespecific effects could in part be responsible for the results. After all, SOL is a slow muscle and less likely to play a role in training of fast lower-leg movements. It is conceivable that the influence of training on the GM might be different from that of the SOL muscle. However, GM EMG was found not to be more active than SOL EMG during the times when H-reflexes were elicited. In line with this, we did not observe a selective GM response, either in the pre- or in post-training measurements. Neither the $\mathrm{M}_{\mathrm{tnax}}$ of the GM nor the GM background EMG activity was changed following training. Most importantly, analysis of the conditioning effects in the GM revealed very similar results to those obtained for the SOL. Therefore, these results are considered a strong indicator that training influenced SOL and GM similarly and it seems unlikely that a differential activation of fast and slow muscles by training or by task, e.g. preferential activation of GM over SOL, would play a role for modulation of SOL $\mathrm{H}_{\text {cond }}$ facilitation.

\section{Lack of spinal effects}

Previous studies on neural adaptation have assessed reflex gain as an estimate of spinal motoneuron excitability, assuming that differences in $\mathrm{H}_{\max } / \mathrm{M}_{\max }$ ratios between untrained and well-trained subjects were due to adaptive effects within the Ia afferent system (for review see
Zehr, 2002). It is generally accepted that presynaptic inhibition of Ia afferents is a major contributor to the regulation of $\mathrm{H}$-reflex gain and it has been shown that presynaptic inhibition is modulated in a taskrelated manner, e.g. increased during free standing as compared to standing with support, or rhythmically modulated during locomotion (Katz et al., 1988; Faist et al., 1996). In humans, there is evidence that presynaptic inhibition and H-reflex gain are under supraspinal control during voluntary movements (Hultborn et al., 1987; Meunier \& Pierrot-Deseilligny, 1989; Nielsen \& Kagamihara, 1993; Perez et al., 2005). Animal studies have shown that supraspinal control of presynaptic inhibition via the pyramidal tract is one relevant mechanism for preserving training-induced plasticity (Wolpaw \& Lee, 1989; Chen \& Wolpaw, 2002). It was therefore expected that H-reflex size would be changed by training, e.g. as an effect of modulation of presynaptic inhibition of la afferents.

In line with other studies (Katz et al., 1988; Faist et al., 1996), the current results show a task-dependency of H-reflex size on posture and activity level. However, training did not induce systematic changes in the $\mathrm{H}_{\max } / \mathrm{M}_{\max }$ ratios. Irrespective of whether the tests were performed at rest or in the active state, there were no significant group effects. This may be due in part to the fact that spinal training effects such as presynaptic control of la afferents in relation to acquisition of a novel motor skill may be short-lasting (10 $\mathrm{min}$ in Perez et al., 2005). Another possible explanation could be underpowered statistical testing, as numbers of included subjects per group were low. As a matter of fact, in two other studies with the same study design significant $\mathrm{H}^{-}$and stretch-reflex reductions were observed after SMT (Gruber et al., 2007b; Taube et al., 2007). Nevertheless, the decrease in $\mathrm{H}_{\max } / \mathrm{M}_{\max }$ ratio in these studies was also explained by supraspinal and not by spinal effects. Therefore, we conclude that the impact of spinal effects on the current findings is comparatively minor.

\section{Functional implications}

In previous studies, short-latency facilitation was shown to be particularly strong during dynamic motor tasks as opposed to tonic activation (Iles \& Pisini, 1992; Nielsen et al., 1993b; Petersen et al., 1998). It may therefore be argued that training effects emphasizing fast activation of SOL are likely to be reflected in changes in short-latency facilitation of $\mathbf{H}_{\text {cond }}$. However, why improved motor performance was associated with a reduction in facilitation needs to be explained. From carlier studies on cortical activation it is known that skilled compared to nonskilled subjects show reduced neural activation in primary and secondary motor areas when performing the same motor action (Jancke et al., 2000; Lotze et al., 2003b; Haslinger et al., 2004). This finding has been interpreted as reffecting diminished neural effort required for a particular motor performance following intensive motor training. There is now increasing knowledge about how short-term training effects are translated into long-term plasticity. Semmler and Nordstrom (1998) assumed that an increase in precision and automaticity during training is associated with a shifting of neural activation from higher (e.g. cortical) sites to 'subordinate' levels with fewer degrees of freedom. Recent findings from functional imaging studies yield proof of this concept, revealing a sequential shift of activation from prefrontal motor cortical areas to primary motor area in an intermediate training phase and, finally, to the basal ganglia and cerebellum during the final stage of automatic movement performance (Floyer-Lea \& Matthews, 2004; Puttemans et al., 2005). This is in line with the present findings of decreased short-latency facilitation after 4 weeks of training indicating reduced corticospinal excitability in the 
trained task. This may be the result of increased motor efficacy and automatic movement control. Conversely, after training, an increase in short-latency facilitation during the nonpractised task (PFL after SMT; PER after BST) would correspond to a raised likelihood of immediately up-regulating corticospinal excitability during unfamiliar tasks which cannot be performed automatically.

Recently, the first successful attempts to treat pharmacologically the lesioned corticospinal tract axons in primates have been reported (Fouad et al., 2004). Despite these promising advances, regeneration will always be incomplete. As is known from animal studies on the upper (Schmidlin et al., 2004) and lower (Bretzner \& Drew, 2005) extremity, the pathways conveying signals from the motor cortex to the spinal motoneurons must undergo reorganization to accomplish compensation for functional deficits following a neural lesion. These observations indicate that the corticospinal tract fibers spared by the lesion are not necessarily sufficient to restore motor control. Thus, plasticity of the remaining intact corticospinal axons is crucial and determinant for capacity to regain motor function.

\section{Acknowledgements}

We thank Dr Franz Aiple, Tom Günther, Florian Pfister and Frank Huethe for technical assistance. This work was supported by the Deutsche Forschungsgemeinschaft (SCHU 1487/1-2).

\section{Abbreviations}

BST, ballistic strength training; CON, control group; EEG, electroencephalogram; EMG, electromyogram; GM, gastrocnemius medialis muscle; $H_{\text {cond }}$ conditioned $\mathrm{H}$-reflex; $\mathrm{H}_{\max }$, maximum $\mathrm{H}$-reflex; ISI, interstimulus interval; MEP, motor evoked potential; $M_{\text {max }}$ maximum $M$-wave; MT, motor threshold; PER, backward stance perturbation; PFL, plantar flexion; REST, rest condition, sitting position; $\mathrm{RFD}_{\max }$, maximum rate of force development; SMT, sensorimotor training, balance training; SOL, soleus muscle; STANCE, standing position; TA, anterior tibial muscle; TMS, transcranial magnetic stimulation.

\section{References}

Aagaard, P., Simonsen, E.B., Andersen, J.L., Magnusson, P. \& Dyhre-Poulsen, P. (2002a) Increased rate of force development and neural drive of human skeletal muscle following resistance training. J. Appl. Physiol., 93, 1318 1326.

Aagaard, P., Simonsen, E.B., Andersen, J.L., Magnusson, P. \& Dyhre-Poulsen, P. (2002b) Neural adaptation to resistance training: changes in evoked V-wave and H-reflex responses. J. Appl. Physiol., 92, 2309-2318.

Beck, S., Taube, W., Gruber, M., Amtage, F., Gollhofer, A. \& Schubert, M. (2007) Task-specific changes in motor evoked potentials of lower limb muscles after different training interventions. Brain Res., 1179, 51-60.

Bretzner, F. \& Drew, T. (2005) Changes in corticospinal efficacy contribute to the locomotor plasticity observed after unilateral cutaneous denervation of the hindpaw in the cat. J. Neurophysiol, 94, 2911-2927.

Carroll, T.J., Rick, S. \& Carson, R.G. (2002) The sites of neural adaptation induced by resistance training in humans. $J$. Physiol. (Lond.), 544, 641-652.

Chen, X.Y. \& Wolpaw, J.R. (2002) Probable corticospinal tract control of spinal cord plasticity in the rat. $J$. Neurophysiol., 87, 645-652.

Classen, J., Liepert, J., Wise, S.P., Hallett, M. \& Cohen, L.G. (1998) Rapid plasticity of human cortical movement representation induced by practice. J. Neurophysiol., 79, 1117-1123.

Crone, C., Hultborn, H., Mazieres, L., Morin, C., Nielsen, J. \& PierrotDeseilligny, E. (1990) Sensitivity of monosynaptic test reflexes to facilitation and inhibition as a function of the test reflex size: a study in man and the cat. Exp. Brain Res., 81, 35-45.

Duchateau, J. \& Hainaut, K. (1984) Isometric or dynamic training: differential effects on mechanical properties of a human muscle. J. Appl. Physiol, 56 , 296-301.

Enoka, R.M. (1997) Neural adaptations with chronic physical activity. J. Biomech., 30, 447-455.
Faist, M., Dictz, V. \& Picrrot-Deseilligny, E. (1996) Modulation, probably presynaptic in origin, of monosynaptic la excitation during human gait. Exp. Brain Res, 109, $441-449$.

Floyer-Lea, A. \& Matthews, P.M. (2004) Changing brain networks for visuomotor control with increased movement automaticity. $\int$. Neurophysiol., 92, 2405-2412.

Fouad, K., Klusman, I. \& Schwab, M.E. (2004) Regenerating corticospinal fibers in the Marmoset (Callitrix jacchus) after spinal cord lesion and treatment with the anti-Nogo-A antibody IN-I. Eur. J. Neurosci., 20, 2479 2482.

Gruber, M. \& Gollhofer, A. (2004) Impact of sensorimotor training on the rate of force development and neural activation. Eur. J. Appl. Physiol., 92, 98-105.

Gruber, M., Gruber, S.B., Taube, W., Schubert, M., Beck, S.C. \& Gollhofer, A (2007a) Differential effects of ballistic versus sensorimotor training on rate of force development and neural activation in humans. $J$. Strength Cond Res., 21, 274-282.

Gruber, M., Taube, W., Gollhofer, A., Beck, S., Amtage, F. \& Schubert, M (2007b) Training-specific adaptations of $\mathrm{H}$ - and stretch reflexes in human soleus muscle. J. Mot. Behav, 39, 68-78.

Hakkinen, K. \& Komi, P.V. (1983) Alterations of mechanical characteristics of human skeletal muscle during strength training. Eur: $J$. Appl. Physiol. Occup Physiol., 50, 161-172.

Haslinger, B., Erhard, P., Altenmuller, E., Hennenlotter, A., Schwaiger, M., von Grafin, E.H., Rummeny, E., Conrad, B. \& Ceballos-Baumann, A.O. (2004) Reduced recruitment of motor association areas during bimanual coordination in concert pianists. Hum. Brain Mapp., 22, 206-215.

Horstmann, G.A., Huethe, F. \& Dietz, V. (1987) [Special treadmill for the investigation of standing and walking in research and in clinical medicine]. Biomed. Techn. (Berl.), 32, 250-254.

Hultborn, H., Meunier, S., Pierrot-Deseilligny, E. \& Shindo, M. (1987) Changes in presynaptic inhibition of la fibres at the onset of voluntary contraction in man. J. Physiol. (Lond.), 389, 757-772.

lles, J.F. \& Pisini, J.V. (1992) Cortical modulation of transmission in spinal reflex pathways of man. $J$. Physiol. (Lond.), 455, 425-446.

Jancke, L., Himmelbach, M., Shah, N.J. \& Zilles, K. (2000) The effect of switching between sequential and repetitive movements on cortical activation. Neuroimage, 12, 528-537.

Jankowska, E., Padel, Y. \& Tanaka, R. (1975) Projections of pyranidal tract cells to alpha-motoneurones innervating hind-limb muscles in the monkey. J. Physiol. (Lond.), 249, 637-667.

Jensen, J.L., Marstrand, P.C. \& Nielsen, J.B. (2005) Motor skill training and strength training are associated with different plastic changes in the centra nervous system. J. Appl. Physiol., 99, 1558-1568.

Katz, R., Meunier, S. \& Pierrot-Deseilligny, E. (1988) Changes in presynaptic inhibition of Ia fibres in man while standing. Brain, 111, 417-437.

Lotze, M., Braun, C., Birbaumer, N., Anders, S. \& Cohen, L.G. (2003a) Motor learning elicited by voluntary drive. Brain, 126, 866 872

Lotze, M., Scheler, G., Tan, H.R., Braun, C. \& Birbaumer, N. (2003b) The musician's brain: functional imaging of amateurs and professionals during performance and imagery. Neuroimage, 20, 1817-1829.

Meunier, S., Kwon, J., Russmann, H., Ravindran, S., Mazzocchio, R. \& Cohen, L. (2007a) Spinal use-dependent plasticity of synaptic transmis sion in humans after a single cycling session. J. Physiol. (Lond.), 579, 375-388.

Meunier, S. \& Pierrot-Deseilligny, E. (1989) Gating of the afferent volley of the monosynaptic stretch refiex during novement in man. $J$. Physiol. (Lond.), 419, 753-763.

Meunier, S., Russmann, H., Simonetta-Moreau, M. \& Hallett, M. (2007b) Changes in Spinal Excitability After PAS. J. Neurophysiol, 97, 31313135 .

Muellbacher, W., Ziemann, U., Boroojerdi, B., Cohen, L. \& Hallett, M. (2001) Role of the human motor cortex in rapid motor learning. Exp. Brain Res., 136, $431-438$.

Muellbacher, W., Ziemann, U., Wissel, J., Dang, N., Kofler, M., Facchini, S., Boroojerdi, B., Poewe, W. \& Hallett, M. (2002) Early consolidation in human primary motor cortex. Nature, 415, 640-644.

Nielsen, J., Crone, C. \& Hultborn, H. (1993a) H-reflexes are smaller in dancers from The Royal Danish Ballet than in well-trained athletes. Eur. J. Appl. Physiol. Occup. Physiol., 66, 116-121.

Nielsen, J. \& Kagamihara, Y. (1993) The regulation of presynaptic inhibition during co-contraction of antagonistic muscles in man. J. Physiol. (Lond.), $464,575-593$

Nielsen, J. \& Petersen, N. (1994) Is presynaptic inhibition distributed to corticospinal fibres in man? J. Physiol. (Lond.), 477, 47-58. 
Nielsen, J. \& Petersen, N. (1995) Changes in the effect of magnetic brain stimulation accompanying voluntary dynamic contraction in man. J. Physiol. (Lond.), 484, 777-789.

Nielsen, J., Petersen, N., Deuschl, G. \& Ballegaard, M. (1993b) Task-related changes in the effect of magnetic brain stimulation on spinal neurones in man. J. Physiol. (Lond.), 471, 223-243.

Pascual-Lcone, A., Grafman, J. \& Hallett, M. (1994) Modulation of cortical motor output maps during development of implicit and explicit knowledge. Science, 263, 1287-1289.

Pascual-Leone, A., Nguyet, D., Cohen, L.G., Brasil-Neto, J.P., Cammarota, A. \& Hallett, M. (1995) Modulation of muscle responses evoked by transcranial magnetic stimulation during the acquisition of new fine motor skills. J. Neurophysiol., 74, 1037-1045.

Perez, M.A., Lundbye-Jensen, J. \& Nielsen, J.B. (2006) Changes in corticospinal drive to spinal motoneurones following visuo-motor skill learning in humans. J. Physiol. (Lond.), 573, 843-855.

Perez, M.A., Lungholt, B.K. \& Nielsen, J.B. (2005) Presynaptic control of group Ia afferents in relation to acquisition of a visuo-motor skill in healthy humans. J. Physiol. (Lond.), 568, 343-354.

Perez, M.A., Lungholt, B.K., Nyborg, K. \& Nielsen, J.B. (2004) Motor skill training induces changes in the excitability of the leg cortical area in healthy humans. Exp. Brain Res., 159, 197-205.

Petersen, N., Christensen, L.O. \& Nielsen, J. (1998) The effect of transcranial magnetic stimulation on the soleus $\mathrm{H}$ reflex during human walking. J. Physiol. (Lond.), 513, 599-610.

Petersen, N.T., Pyndt, H.S. \& Nielsen, J.B. (2003) Investigating human motor control by transcranial magnetic stimulation. Exp. Brain Res., 152, 1-16.

Puttemans, V., Wenderoth, N. \& Swinnen, S.P. (2005) Changes in brain activation during the acquisition of a multifrequency bimanual coordination task: from the cognitive stage to advanced levels of automaticity. J. Neurosci., 25, 4270-4278.

Remple, M.S., Bruneau, R.M., VandenBerg, P.M., Goertzen, C. \& Kleim, J.A. (2001) Sensitivity of cortical movement representations to motor experience: evidence that skill leaming but not strength training induces cortical reorganization. Behav. Brain Res., 123, 133-141.

Schmidlin, E., Wannier, T., Bloch, J. \& Rouiller, E.M. (2004) Progressive plastic changes in the hand representation of the primary motor cortex parallel incomplete recovery from a unilateral section of the corticospinal tract at cervical level in monkeys. Brain Res., 1017, 172-183.

Schubert, M., Curt, A., Colombo, G., Berger, W. \& Dietz, V. (1999) Voluntary control of human gait: conditioning of magnetically evoked motor responses in a precision stepping task. Exp. Brain Res, 126, 583-588.

Schubert, M., Curt, A., Jensen, L. \& Dietz, V. (1997) Corticospinal input in human gait: modulation of magnetically evoked motor responses. Exp. Brain Res., 115, 234-246.

Semmler, J.G. \& Nordstrom, M.A. (1998) Motor unit discharge and force tremor in skill- and strength-trained individuals. Exp. Brain Res., 119, 27-38.

Taube, W., Gruber, M., Beck, S., Faist, M., Gollhofer, A. \& Schubert, M. (2007) Cortical and spinal adaptations induced by balance training: correlation between stance stability and corticospinal activation. Acta Physiol. (Oxf.), 189, 347-358.

Taube, W., Schubert, M., Gruber, M., Beck, S., Faist, M. \& Gollhofer, A. (2006) Direct corticospinal pathways contribute to neuromuscular control of perturbed stance. J. Appl. Physiol., 101, 420-429.

Van Cutsem, M., Duchateau, J. \& Hainaut, K. (1998) Changes in single motor unit behaviour contribute to the increase in contraction speed after dynamic training in humans. J. Physiol. (Lond.), 513, 295-305.

Voigt, M., Chelli, F. \& Frigo, C. (1998) Changes in the excitability of soleus muscle short latency stretch reflexes during human hopping after 4 weeks of hopping training. Eut: J. Appl. Physiol. Occup. Physiol, 78, $522-532$.

Wolpaw, J.R. \& Lee, C.L. (1989) Memory traces in primate spinal cord produced by operant conditioning of H-reflex. $J$. Neurophysiol., 61, 563-572.

Zehr, E.P. (2002) Considerations for use of the Hoffmann reflex in exercise studies. Eur: J. Appl. Physiol., 86, 455-468. 